\title{
PRESERVATION OF MYOCYTE CONTRACTILE FUNCTION AFTER HYPOTHERMIC, HYPERKALEMIC CARDIOPLEGIC ARREST WITH 2,3-BUTANEDIONE MONOXIME
}

\author{
B. Hugh Dorman, $\mathrm{MD}, \mathrm{PhD}$ \\ Martyn J. Cavallo, MD \\ Robert B. Hinton, BA \\ Raymond C. Roy, $\mathrm{MD}, \mathrm{PhD}$ \\ Francis G. Spinale, MD, $\mathrm{PhD}$
}

One proposed contributory mechanism for depressed ventricular performance after hypothermic, hyperkalemic cardioplegic arrest is a reduction in myocyte contractile function caused by alterations in intracellular calcium homeostasis. Because 2,3-butanedione monoxime decreases intracellular calcium transients, this study tested the hypothesis that 2,3 butanedione monoxime supplementation of the hyperkalemic cardioplegic solution could preserve isolated myocyte contractile function after hypothermic, hyperkalemic cardioplegic arrest. Myocytes were isolated from the left ventricles of six pigs. Magnitude and velocity of myocyte shortening were measured after 2 hours of incubation under normothermic conditions $\left(37^{\circ} \mathrm{C}\right.$, standard medium), hypothermic, hyperkalemic cardioplegic arrest ( $4^{\circ} \mathrm{C}$ in Ringer's solution with $20 \mathrm{mEq}$ potassium chloride), and hypothermic, hyperkalemic cardioplegic arrest with 2,3-butanedione monoxime supplementation ( $4^{\circ} \mathrm{C}$ in Ringer's solution with $20 \mathrm{mEq}$ potassium chloride and $20 \mathrm{mmol} / \mathrm{L} \mathrm{2,3-butanedione} \mathrm{monoxime).} \mathrm{Because} \beta$-adrenergic agonists are commonly employed after cardioplegic arrest, myocyte contractile function was examined in the presence of the $\beta$-agonist isoproterenol ( 25 nmol/L). Hypothermic, hyperkalemic cardioplegic arrest and rewarming reduced the velocity $(32 \%)$ and percentage of myocyte shortening $(27 \%, p<$ 0.05). Supplementation with 2,3-butanedione monoxime normalized myocyte contractile function after hypothermic, hyperkalemic cardioplegic arrest. Although $\beta$-adrenergic stimulation significantly increased myocyte contractile function under normothermic conditions and after hypothermic, hyperkalemic cardioplegic arrest, contractile function of myocytes exposed to $\boldsymbol{\beta}$-agonist after hypothermic, hyperkalemic cardioplegic arrest remained significantly reduced relative to the normothermic control group. Supplementation with 2,3-butanedione monoxime restored $\beta$-adrenergic responsiveness of myocytes after hypothermic, hyperkalemic cardioplegic arrest. Thus, supplementation of a hyperkalemic cardioplegic solution with 2,3-butanedione monoxime had direct and beneficial effects on myocyte contractile function and $\boldsymbol{\beta}$-adrenergic responsiveness after cardioplegic arrest. A potential mechanism for the effects of 2,3-butanedione monoxime includes modulation of intracellular calcium transients or alterations in sensitivity to calcium. Supplementation with 2,3 -butanedione monoxime may have clinical utility in improving myocardial contractile function after hypothermic, hyperkalemic cardioplegic arrest. (J THORAC CARDIOvasc SURG 1996;111:621-9)
From the Medical University of South Carolina, Departments of Anesthesia and Perioperative Medicine and Cardiothoracic Surgery, Charleston, S.C.

Supported by National Institutes of Health Grant R29-HL-45024, a basic research grant from Pfizer, Inc., MUSC postdoctoral research award (M.J.C.), and South Carolina American Heart Association (B.H.D.). F.G.S. is an established investigator of the American Heart Association.
Received for publication April 4, 1995; accepted for publication June 12, 1995.

Address for reprints: B. Hugh Dorman, MD, PhD, Medical University of South Carolina, Department of Anesthesiology, 171 Ashley Ave., Charleston, SC 29425-2207.

Copyright (C) 1996 by Mosby-Year Book, Inc.

$0022-5223 / 96 \$ 5.00+0 \quad \mathbf{1 2 / 1 / 6 7 0 3 4}$ 
$\mathrm{M}$ yocardial preservation during cardiopulmonary bypass for cardiac surgery has traditionally involved the use of hypothermic, hyperkalemic cardioplegia. ${ }^{1}$ The fundamental basis for the clinical use of hyperkalemic cardioplegia is that it causes rapid membrane depolarization and produces a quiescent heart. ${ }^{2}$ This membrane depolarization in the presence of elevated extracellular potassium concentrations results in a cessation of contractile activity, thereby reducing metabolic demands within the myocyte. $^{3,4}$ Extracellular hyperkalemia has significant effects on ionic homeostasis, however, and causes an intracellular calcium overload state that, with reperfusion, has been shown to result in alterations in contractile performance and abnormal regulation of intracellular enzyme systems and volume regulatory mechanisms. ${ }^{5-10}$ Furthermore, any myocyte ischemia during cardioplegic arrest contributes to the increased intracellular concentration of free calcium and myocyte damage. ${ }^{11-14}$ Because increases in intracellular calcium associated with hyperkalemic cardioplegic arrest appear to play a pivotal role in myocyte contractile dysfunction and derangements in metabolic processes, prevention of an elevated intracellular calcium state during hyperkalemic cardioplegic arrest may preserve myocyte contractile function.

The agent 2,3-butanedione monoxime (BDM) has the ability to uncouple cardiac excitation from contraction, prolong myocardial ischemic tolerance, and protect ischemic myocardium from calciuminduced adenosine triphosphate (ATP) loss and the damaging effects of ischemic contracture. ${ }^{15,16} \mathrm{Al}$ though the exact mechanism by which BDM affects contractile activity is not completely understood, in part BDM appears to cause a reduction in myofibrillar sensitivity to calcium. ${ }^{17-19}$ Moreover, BDM has been shown to decrease intracellular calcium transients secondary to a reduction in the release of calcium from the sarcoplasmic reticulum and a reduction in the transsarcolemmal calcium flux. ${ }^{18,20-22}$ BDM-induced decreases in myocyte calcium transients and myofibrillar sensitivity to calcium may prove useful in attenuating the derangements in myocyte contractile function and metabolic regulatory processes associated with a calcium overload state after hyperkalemic cardioplegic arrest. Our purpose in this study was therefore to examine the effects of BDM supplementation of hyperkalemic cardioplegia on myocyte contractile function and cellular swelling in an isolated myocyte model of hypothermic, hyperkalemic cardioplegic arrest to better understand both optimal myocardial preservation and contractile dysfunction associated with hyperkalemic cardioplegic arrest.

\section{Methods}

Myocyte isolation and contraction. Myocyte isolation and measurement of myocyte contractile function were performed according to previously described methods, well characterized by reports from this laboratory. $8,9,23$ Yorkshire swine were the source of myocytes used in this study. All animals were cared for and treated in accordance with the "Guide for the Care and Use of Laboratory Animals" prepared by the Institute of Laboratory Animal Resources and published by the National Institutes of Health (NIH Publication No. 86-23, revised 1985). Surgical anesthesia was provided with $2 \%$ isoflurane in oxygen, and the heart was quickly removed and placed in an oxygenated Krebs solution. A portion of the left ventricular free wall $(5 \times 5 \mathrm{~cm})$ containing the circumflex artery was excised and used for myocyte isolation. After cannulation of the circumflex artery and ligation of distal branches, the tissue was rinsed free of blood with $30 \mathrm{ml}$ modified Krebs solution containing $5 \mathrm{mmol} / \mathrm{L}$ nitrilotriacetic acid and $0.1 \%$ bovine serum albumin. Collagenase $(0.5 \mathrm{mg} / \mathrm{ml}$, Worthington Biochemical Corp., Freehold, N.J.; type II, $146 \mathrm{U} / \mathrm{mg}$ ) was added to the solution and the myocardium was perfused for 35 minutes. The tissue was then minced into $2 \mathrm{~mm}$ sections and added to an oxygenated solution containing $2 \%$ bovine serum albumin, deoxyribonuclease II (DNase, 51 Kunitz units/ml, type IV; Sigma Chemical Co., St. Louis, Mo.), $300 \mu \mathrm{mol} / \mathrm{L}$ calcium chloride, and $0.5 \mathrm{mg} / \mathrm{ml}$ collagenase. The supernatant was removed and the myocyte pellet was resuspended in standard cell culture medium $\left(2 \mathrm{mmol} / \mathrm{L} \mathrm{Ca}^{2+}\right.$, medium M199; Life Technologies, Gaithersburg, Md.). A $2 \mathrm{ml}$ aliquot of the isolated myocyte suspension was then plated onto coverslips coated with a basement membrane substrate (Matrigel; Collaborative Biomedical Inc., Bedford, Mass.), stabilized at $37^{\circ} \mathrm{C}$ in oxygenated medium for 60 minutes, and then randomly assigned to treatment protocols after measurement of baseline contractile function. Viable myocytes included those that retained a rod shape, were calcium tolerant, and excluded trypan blue dye. The percentage of viable myocytes averaged $80 \% \pm 3 \%$, with no significant differences between treatment groups.

For contractile function measurements, myocytes were placed in a thermostatically controlled chamber containing standard cell culture medium $\left(37^{\circ} \mathrm{C}\right)$ for imaging on an inverted microscope (Axiovert IM35; Carl Zeiss, Oberkochen, Germany). The chamber $(2.5 \mathrm{ml})$ contained two stimulating platinum electrodes and a miniature thermocouple (CN7100; Omega Engineering, Inc., Stamford, Conn.). Imaging of myocytes was accomplished with a $\times 20$ Hoffman Modulation Contrast Objective (Modulation Optics, Inc., Greenvale, N.Y.) with a final magnification of $\times 1100$. Myocyte contractions were elicited by field stimulating the tissue chamber at $1 \mathrm{~Hz}$ (S11; Grass Instrument Co., Quincy, Mass.) with current pulses of 5 msec duration and voltages $10 \%$ above contraction threshold. A standard voltage (3 volts) was delivered to the medium for all myocytes studied. Polarity of the stimulating electrodes was alternated at every pulse to 
prevent accumulation of electrochemical byproducts. Myocyte contractions were imaged with a charge-coupled device with a noninterlaced scan rate of $240 \mathrm{~Hz}$ (GPCD 60; Panasonic Co., Secaucus, N.J.). Myocyte motion signals were captured with the cell parallel to the video raster lines, and this video signal was input through an edgedetector system (Crescent Electronics, Sandy, Utah). Changes in light intensity at the myocyte edges were used to track myocyte motion. The distance between the left and right myocyte edges was converted into a voltage signal, digitized, and input to a computer (80286, ZBV2526; Zenith Data Systems, St. Joseph, Mich.) for subsequent analysis. Stimulated myocytes were allowed a 5 -minute stabilization period after electrical stimulation, and contraction data for each myocyte were recorded from a minimum of 20 consecutive contractions. Parameters computed from the digitized contraction profiles included the following: magnitude of shortening (expressed as a percentage), velocity of shortening (in micrometers per second), velocity of relengthening (in micrometers per second), time to $50 \%$ relaxation (in milliseconds), total contraction duration (in milliseconds), and time to peak contraction (in milliseconds). Myocyte percentage shortening was determined as the percentage difference between maximum and minimum cell length for each contraction. Myocyte shortening velocity computations were obtained by differentiating the digitized contraction profiles. The time to peak contraction was computed by calculating the time required for the differentiated velocity profile to reach zero velocity after the start of contraction. All parameters were calculated for each contraction and the results were averaged for the 20 contractions observed.

Cardioplegia and drug administration. After determination of initial myocyte contractile function, myocytes were randomly assigned to one of three treatment protocols. (1) In the normothermic control group, the following protocol was performed: Myocytes were incubated at $37^{\circ} \mathrm{C}$ in Ringer's solution containing $30 \mathrm{mmol} / \mathrm{L}$ sodium

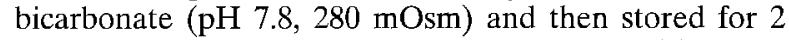
hours at $37^{\circ} \mathrm{C}$ in a $95 \%$ oxygen environment. (2) In the cardioplegia group, the following protocol was performed: Myocytes were incubated at $4^{\circ} \mathrm{C}$ in Ringer's solution containing $24 \mathrm{mEq} / \mathrm{L}$ potassium chloride and $30 \mathrm{mEq} / \mathrm{L}$ sodium bicarbonate ( $315 \mathrm{mOsm}$ ), then stored at $4^{\circ} \mathrm{C}$ for 2 hours with subsequent rewarming for 10 minutes. (3) In the BDM-cardioplegia group, the following protocol was performed: Myocytes were incubated at $4^{\circ} \mathrm{C}$ in Ringer's solution containing $24 \mathrm{mEq} / \mathrm{L}$ potassium chloride, 30 $\mathrm{mEq} / \mathrm{L}$ sodium bicarbonate, and $20 \mathrm{mmol} / \mathrm{L}$ BDM (453 mOsm), then stored at $4^{\circ} \mathrm{C}$ for 2 hours with subsequent rewarming in standard cell culture medium for $10 \mathrm{~min}$ utes. This concentration of BDM $(20 \mathrm{mmol} / \mathrm{L})$ was chosen because it has been shown to inhibit force development in cultured ventricular myocytes and to improve myocardial ischemic tolerance without adverse effects on isolated myocyte viability. 15,24

After the respective treatment protocol and rewarming, myocytes were transferred to the stimulation chamber, electrical stimulation was initiated, and steady-state contractile function measurements were obtained as described in the previous section. Because initial pretreat- ment myocyte contractile function did not differ significantly from myocyte contractile function in the normothermic control group, only values for the normothermic control group are reported. After measurement of contractile function, myocytes were exposed to $25 \mathrm{nmol} / \mathrm{L}$ isoproterenol and measurements of contractile function were repeated. The oxygen tension of the incubation medium did not differ significantly between treatment groups at the beginning or end of the experimental protocol and ranged between 210 and $270 \mathrm{~mm} \mathrm{Hg}$.

Myocyte dimensions. Resting myocyte length $(\mu \mathrm{m})$ was measured under normothermic control conditions and after hypothermic, hyperkalemic cardioplegic arrest with and without BDM supplementation of the cardioplegia solution. To examine more carefully the issue of myocyte swelling after cardioplegic arrest, myocytes from the normothermic control group, cardioplegia group, and BDMcardioplegia group were immediately placed in a buffered sodium cacodylate solution containing $2 \%$ paraformaldehyde and $2 \%$ glutaraldehyde ( $\mathrm{pH} 7.4,325 \mathrm{mOsm}$ ). The isolated myocytes were then imaged with a $\times 10$ phasecontrast objective. The image was input into an imageanalysis system (IBAS 2000 Image Analysis System; Carl Zeiss) and the images were digitized at $512 \times 512$ line resolution and 256 gray levels. Individual cell profiles were automatically discriminated by gray level to determine myocyte profile surface area. ${ }^{25}$

Data analysis. Changes in indexes of myocyte function between the control and cardioplegia groups were examined with multiway analysis of variance. If analysis of variance revealed significant differences, pairwise tests of individual group means were compared with Bonferroni's probabilities. All statistical analyses were performed with standard statistical software programs (BMDP Statistical Software Inc., University of California Press, Los Angeles, Calif.). Results are presented as mean \pm standard error of the mean. Values of $p<0.05$ were considered to be statistically significant.

\section{Results}

Representative contraction profiles at baseline for normothermic control and cardioplegia myocytes are illustrated in Fig. 1, and steady-state myocyte contractile function for these two treatment groups is summarized in Table I. A significant reduction in contractile function was observed for myocytes exposed to hypothermic, hyperkalemic cardioplegic arrest and rewarming. Specifically, isolated myocyte velocity of shortening was reduced by $32 \%$ and myocyte percentage shortening was reduced by $27 \%$ in the cardioplegia group relative to the normothermic control group. The velocity of relengthening, which is an index of active relaxation, was significantly decreased by $33 \%$ in myocytes after hyperkalemic cardioplegic arrest.

To determine the effect of BDM supplementation on myocyte contractile function after hypothermic, hyperkalemic cardioplegic arrest, $20 \mathrm{mmol} / \mathrm{L} \mathrm{BDM}$ 

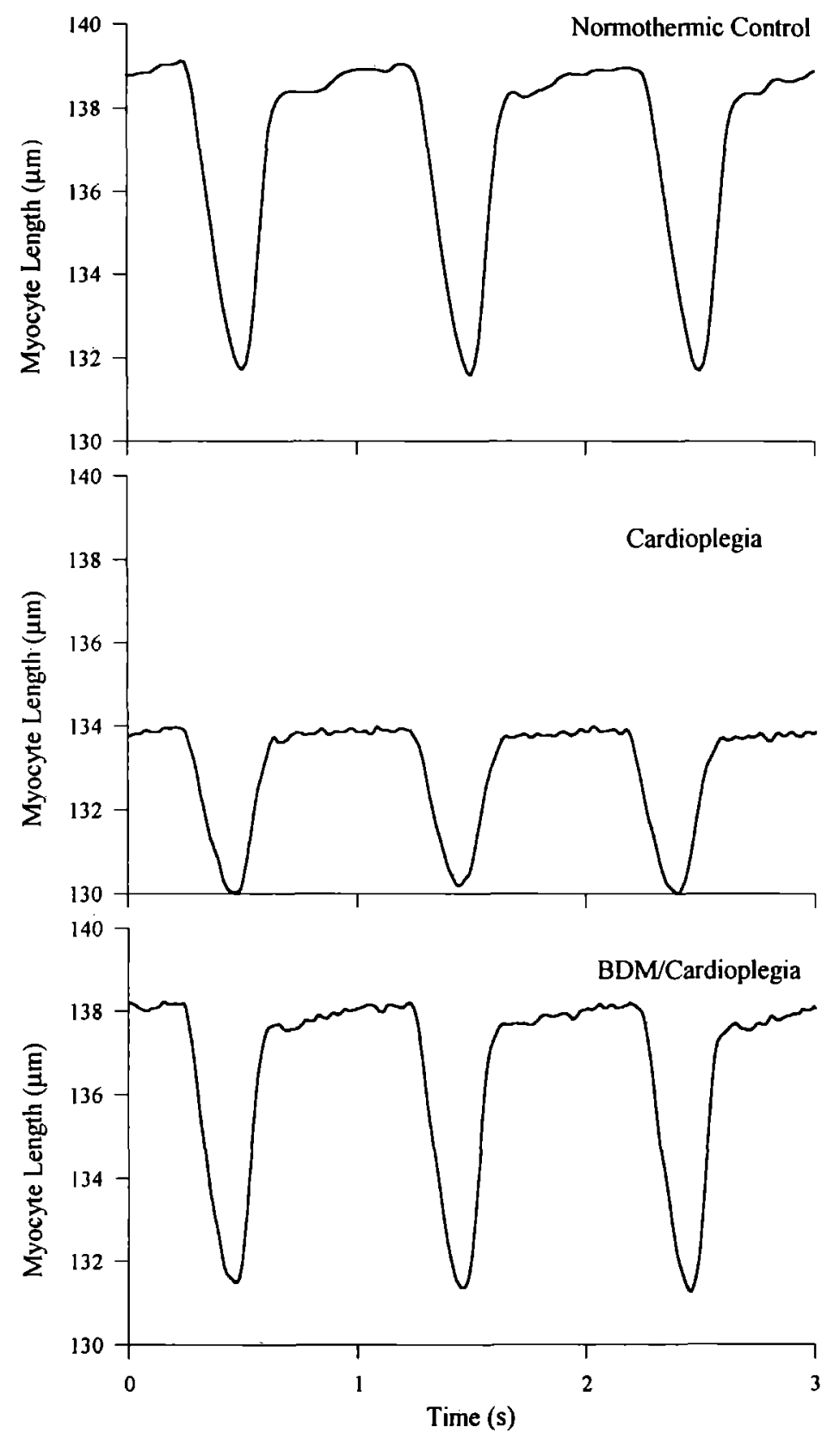

Fig. 1. Representative contraction profiles for myocytes from the normothermic control, cardioplegia, and BDM-cardioplegia treatment groups. The extent of myocyte shortening with each contraction was significantly reduced for myocytes after hyperkalemic cardioplegic arrest. The addition of BDM to the cardioplegia solution restored myocyte shortening after hyperkalemic cardioplegic arrest to normothermic control values.

was included in the standard cardioplegic solution for the 2-hour incubation period. Steady-state myocyte contractile function from this portion of the study is illustrated in Table I. BDM supplementation preserved myocyte contractile function after hypothermic, hyperkalemic cardioplegic arrest.
There were no differences in velocity of shortening, percentage shortening, or velocity of relengthening between myocytes in the BDM-cardioplegia and normothermic control groups. A significant increase in velocity of shortening (21\%) and percentage shortening $(23 \%)$ was observed in myocytes with 
BDM supplementation (BDM-cardioplegia group) relative to myocytes that underwent cardioplegic arrest without BDM supplementation (cardioplegia group). Moreover, a significant increase (62\%) in velocity of relengthening was measured in myocytes in the BDM-cardioplegia group relative to myocytes in the cardioplegia group. The time to $50 \%$ relaxation was significantly reduced in myocytes in the BDM-cardioplegia group relative to both normothermic control and cardioplegia groups.

To determine the effects of BDM supplementation on $\beta$-adrenergic responsiveness after hypothermic, hyperkalemic cardioplegic arrest, $25 \mathrm{nmol} / \mathrm{L}$ isoproterenol was added to the medium of myocytes in the normothermic control, cardioplegia, and BDM-cardioplegia groups. The concentration of 25 $\mathrm{nmol} / \mathrm{L}$ isoproterenol used in this study has been shown to produce a maximal contractile response in normothermic control myocytes. ${ }^{8,9,23}$ The results for $\beta$-adrenergic stimulation are summarized in Table I. Isoproterenol resulted in a significant increase in myocyte contractile function in normothermic control, cardioplegia, and BDM-cardioplegia groups. The velocity of shortening and percentage shortening increased by more than $150 \%$ in all myocyte treatment groups. Contractile function, however, of cardioplegia myocytes after isoproterenol administration remained significantly decreased compared with normothermic control myocytes. Cardioplegia supplementation with BDM significantly improved $\beta$-adrenergic responsiveness after cardioplegic arrest to values observed with normothermic control myocytes. Myocytes exposed to cardioplegic arrest with BDM supplementation displayed significantly increased percentage and velocity of shortening relative to myocytes exposed to cardioplegic arrest without BDM supplementation after isoproterenol administration. Moreover, velocity of relengthening for myocytes after cardioplegic arrest and isoproterenol administration was significantly higher (33\%) when BDM supplementation was used. There were no differences in myocyte contractile indexes between normothermic control and BDM-cardioplegia myocytes after isoproterenol administration, with the exception of a reduced time to peak contraction and decreased total contraction duration observed in myocytes in the BDM-cardioplegia group.

To determine the effect of BDM on myocyte dimensions after hypothermic, hyperkalemic cardioplegic arrest, isolated myocyte profile surface area was measured by means of computer digitiza-
Table I. Effects of BDM supplementation on myocyte contractile function after hypothermic hypervolemic cardioplegic arrest

\begin{tabular}{|c|c|c|}
\hline & Baseline & $\begin{array}{r}\text { Isoproterenol } \\
(25 \mathrm{nmol} / \mathrm{L})\end{array}$ \\
\hline \multicolumn{3}{|l|}{ Resting length $(\mu \mathrm{m})$} \\
\hline Normothermic control & $139 \pm 3$ & $134 \pm 3$ \\
\hline Cardioplegia & $132 \pm 3$ & $128 \pm 3$ \\
\hline BDM-cardioplegia & $134 \pm 3$ & $130 \pm 3$ \\
\hline \multicolumn{3}{|c|}{ Velocity of shortening $(\mu \mathrm{m} / \mathrm{sec})$} \\
\hline Normothermic control & $50 \pm 3$ & $154 \pm 9^{*}$ \\
\hline Cardioplegia & $34 \pm 1 \dagger$ & $130 \pm 5^{*} \dagger$ \\
\hline BDM-cardioplegia & $43 \pm 2 \ddagger$ & $166 \pm 8^{*} \div$ \\
\hline \multicolumn{3}{|l|}{ Shortening $(\%)$} \\
\hline Normothermic control & $4.5 \pm 0.1$ & $9.5 \pm 0.3^{*}$ \\
\hline Cardioplegia & $3.3 \pm 0.1 \uparrow$ & $8.6 \pm 0.3^{*} \dagger$ \\
\hline BDM-cardioplegia & $4.3 \pm 0.2 \ddagger$ & $10.1 \pm 0.4^{*} \ddagger$ \\
\hline \multicolumn{3}{|l|}{$\begin{array}{l}\text { Velocity of relengthening } \\
\qquad(\mu \mathrm{m} / \mathrm{sec})\end{array}$} \\
\hline Normothermic control & $55 \pm 3$ & $139 \pm 9^{*}$ \\
\hline Cardioplegia & $37 \pm 2 \uparrow$ & $115 \pm 6^{* \dagger}$ \\
\hline BDM-cardioplegia & $60 \pm 4 \div$ & $153 \pm 9 * \div$ \\
\hline \multicolumn{3}{|c|}{ Time to $50 \%$ relaxation $(\mathrm{msec})$} \\
\hline Normothermic control & $95 \pm 3$ & $77 \pm 2^{*}$ \\
\hline Cardioplegia & $100 \pm 4$ & $81 \pm 4^{*}$ \\
\hline BDM-cardioplegia & $84 \pm 2 \dagger \ddagger$ & $76 \pm 3^{*}$ \\
\hline $\begin{array}{l}\text { Time to peak contraction } \\
\text { (msec) }\end{array}$ & & - \\
\hline Normothermic control & $266 \pm 7$ & $220 \pm 6^{*}$ \\
\hline Cardioplegia & $255 \pm 5$ & $190 \pm 4 * 广$ \\
\hline BDM-cardioplegia & $259 \pm 5$ & $188 \pm 4^{*} \dagger$ \\
\hline \multicolumn{3}{|l|}{$\begin{array}{l}\text { Total contraction duration } \\
\quad(\mathrm{msec})\end{array}$} \\
\hline Normothermic control & $474 \pm 12$ & $402 \pm 8^{*}$ \\
\hline Cardioplegia & $464 \pm 9$ & $388 \pm 11^{*}$ \\
\hline BDM-cardioplegia & $448 \pm 7$ & $371 \pm 9^{*} \dagger$ \\
\hline \multicolumn{3}{|l|}{ Number of myocytes } \\
\hline Normothermic control & 67 & 64 \\
\hline Cardioplegia & 70 & 68 \\
\hline BDM-cardioplegia & 82 & 78 \\
\hline
\end{tabular}

tion methods for normothermic control myocytes and myocytes after cardioplegic arrest, with and without BDM supplementation. As illustrated in Fig. 2, myocyte profile surface area formed a Gaussian distribution in the normothermic control group, with a mean value of $3445 \pm 47 \mu \mathrm{m}^{2}$. Hypothermic, hyperkalemic cardioplegic arrest resulted in a significant increase in myocyte profile surface area to $4024 \pm 56 \mu \mathrm{m}^{2}(p<0.05)$. BDM supplementation resulted in a significant reduction in myocyte surface area after cardioplegic arrest to $3312 \pm 42 \mu \mathrm{m}^{2}$ $(p<0.05)$, a value comparable with normothermic control myocytes. 

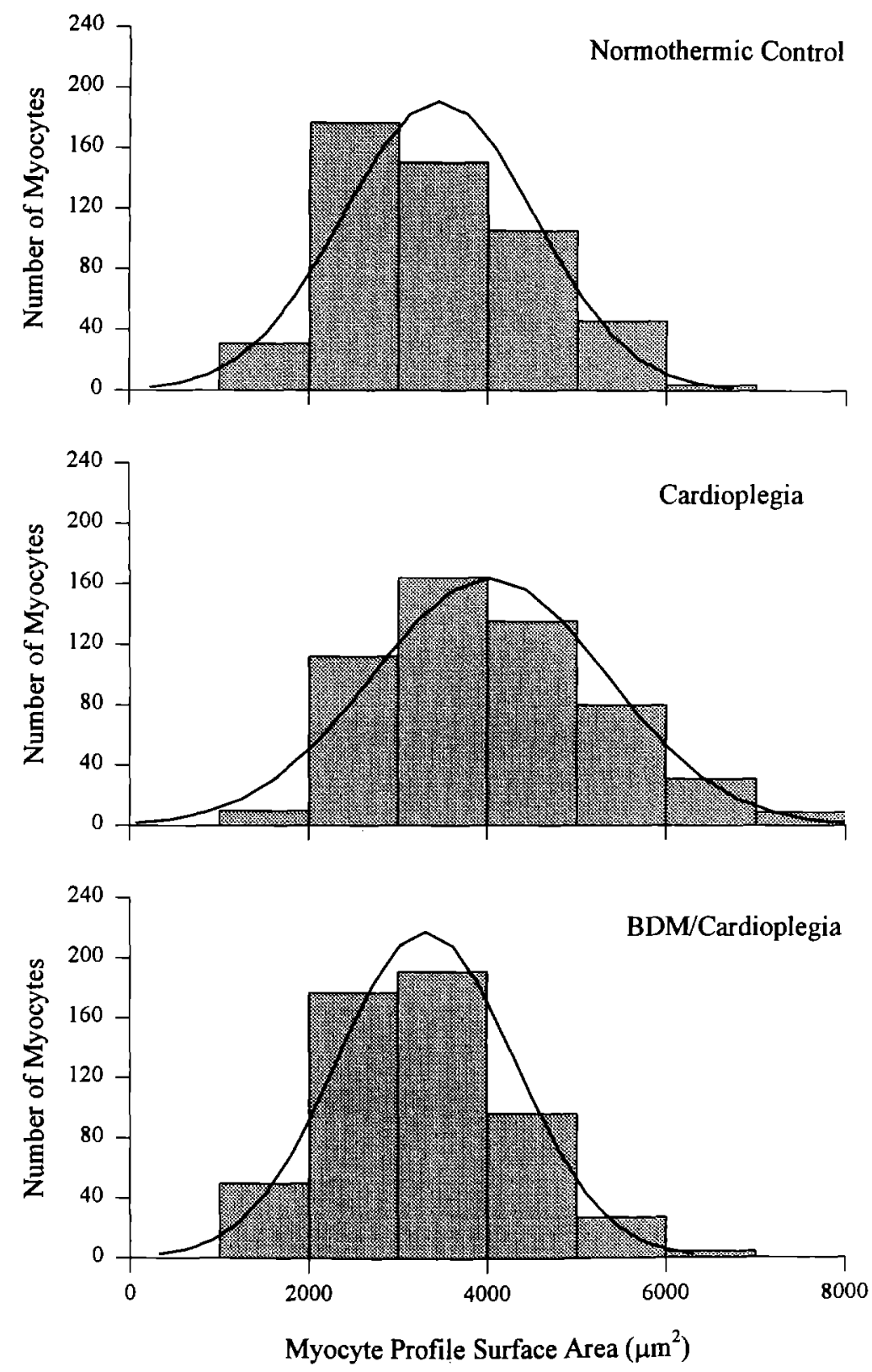

Fig. 2. Profile surface area for myocytes from the normothermic control, cardioplegia, and BDMcardioplegia treatment groups. Profile surface area formed a Gaussian distribution. A significant increase in myocyte profile surface area occurred after hyperkalemic cardioplegic arrest. BDM supplementation of the cardioplegia solution prevented the increase in myocyte profile surface area associated with hyperkalemic cardioplegic arrest.

\section{Discussion}

Despite significant advances in myocardial preservation techniques, a reduction in left ventricular function still occurs after hypothermic, hyperkalemic cardioplegic arrest, and plays a major role in patient morbidity. ${ }^{1}$ The cellular basis and contributory mechanisms involved in such ventricular dys- function after hypothermic, hyperkalemic cardioplegic arrest remain unclear but may involve an excess of intracellular calcium associated with a hyperkalemic extracellular milieu. ${ }^{6,7}$ This study demonstrates for the first time that the deleterious effects of hypothermic, hyperkalemic cardioplegic arrest on contractile function, $\beta$-receptor stimulation, and 
volume regulatory processes within the myocyte can be prevented by the addition of BDM to the hyperkalemic cardioplegia solution.

The mechanism through which BDM preserved isolated myocyte contractile function in this study after hyperkalemic cardioplegic arrest is speculative, but is probably related to prevention of widespread cellular damage of myocytes caused by an overload of intracellular calcium. ${ }^{11,26,27}$ The hyperkalemic environment associated with cardioplegia has been shown to induce an increase in intracellular calcium by several mechanisms, including increased transsarcolemmal calcium flux through slow calcium channels, potentiation of calcium release from the sarcoplasmic reticulum, and increase in calcium influx through sodium-calcium exchange activity. ${ }^{6,7,28-30}$ This calcium overload state during cardioplegic arrest is further enhanced by an increase in cytosolic levels of free calcium that occurs with either ischemia or prolonged metabolic inhibition and contributes to calcium-induced myocyte damage. ${ }^{12-14} \mathrm{BDM}$ has been shown to decrease intracellular calcium transients by reducing the transsarcolemmal calcium flux and decreasing the release of calcium from the sarcoplasmic reticulum. ${ }^{18,20-22}$ Moreover, BDM can result in a reduction in myofibrillar sensitivity to calcium. ${ }^{17-19}$ BDM has also been shown to uncouple myocardial excitation from contraction, presumably by a direct action on myosin molecules, and to protect ischemic myocardium from calcium-stimulated ATP loss and the damaging effects of ischemic contracture. ${ }^{15,16,31}$ There is also evidence that the inhibition of calcium-dependent force development by BDM can protect against reoxygenation hypercontracture in ventricular myocytes. ${ }^{26}$ Such reductions in intracellular free calcium and attenuation of calcium effects may therefore be responsible for the protective effect of BDM on myocyte contractile function after hyperkalemic cardioplegic arrest observed in this study. Direct measurements of intracellular calcium during hyperkalemic cardioplegic arrest and reperfusion with and without BDM supplementation would be useful to determine the contribution of alterations in cytosolic calcium transients to the protective effect observed.

One frequent consequence of hypothermic hyperkalemic cardioplegic arrest is myocardial edema. ${ }^{32}$ With reperfusion after cardioplegic arrest, myocardial water content can increase, resulting in a reduction in left ventricular compliance, which in turn contributes to a deterioration in left ventricular performance..$^{32,33}$ In a previous study by this laboratory, we determined that a significant increase in isolated myocyte profile surface area occurred after hypothermic, hyperkalemic cardioplegic arrest, showing that the intracellular compartment participates in the myocardial edema observed in vivo. 8,32 The increase in myocyte profile surface area after hyperkalemic cardioplegic arrest is indicative of marked cellular swelling, since surface area is directly proportional to myocyte volume. ${ }^{34}$ In the present study, BDM supplementation of the cardioplegia solution prevented the cellular swelling observed after hyperkalemic cardioplegic arrest. These results indicate that the disruption of cell volume regulatory processes associated with hyperkalemic cardioplegic arrest were prevented by the presence of BDM, which may reflect improvement of ionic homeostasis by BDM supplementation during hyperkalemic cardioplegic arrest.

In this study, the velocity of myocyte relengthening, which is an index of active relaxation processes and diastolic function, was significantly reduced after hypothermic hyperkalemic cardioplegic arrest. Increased intracellular calcium concentrations in diastole after reperfusion have been shown to be associated with a significant reduction of ventricular compliance and impaired relaxation, with actinmyosin cross-bridge formation and depletion of ATP levels. ${ }^{35}$ The capacity for the sarcoplasmic reticulum of a postischemic myocyte to participate in the uptake of calcium may be impaired, which would result in increased diastolic intracellular calcium concentrations. ${ }^{36,37}$ The impairment of diastolic myocyte contractile function after hypothermic, hyperkalemic cardioplegic arrest has been shown to be especially pronounced in the presence of elevated extracellular calcium concentration, suggesting a potentiation of intracellular calcium overload in diastole through passive diffusion. ${ }^{8}$ In this study, BDM supplementation restored active relaxation of myocytes after cardioplegic arrest to normothermic control values, which may in part reflect BDM-induced alterations in calcium transients. In fact, the time to $50 \%$ relaxation was reduced in myocytes after cardioplegic arrest with BDM supplementation compared with normothermic control myocytes, which again confirms improved diastolic function. This finding agrees with studies of wholeheart preparations undergoing prolonged hypothermic preservation in which BDM supplementation was shown to improve diastolic relaxation. ${ }^{15,16}$ BDM inhibits actin-myosin cross-bridge formation 
and causes a decrease in myosin ATPase activity, which helps preserve ATP levels during arrest. ${ }^{15,38}$ Because active relaxation is an energy-dependent process, the preservation of ATP levels by BDM may contribute to the improved diastolic function.

An increase in myocyte contractile function was caused by $\beta$-adrenergic stimulation both in the normothermic control group and after hyperkalemic cardioplegic arrest. In agreement with previous studies, however, $\beta$-adrenergic responsiveness was reduced after hyperkalemic cardioplegic arrest. 8,9 The decrease in $\beta$-receptor density, concurrent with desensitization and down regulation of the $\beta$-receptor system that has been shown to occur after hyperkalemic cardioplegic arrest, probably plays a major role in the reduced $\beta$-adrenergic responsiveness observed. ${ }^{39}$ It is also possible that elevated levels of cytosolic calcium during hyperkalemic cardioplegic arrest may have caused intracellular damage and thereby reduced activity downstream from the $\beta$-adrenergic receptor system, affecting phosphorylation and integrity of calcium channels, or affecting actin-myosin complex cross-bridge cycling. ${ }^{40,41}$ BDM supplementation of cardioplegia restored $\beta$-adrenergic responsiveness of myocytes after hyperkalemic cardioplegic arrest, which may in part reflect prevention of calcium-induced cellular damage. ${ }^{11,15,26,42}$ There is also evidence that synergy exists between cyclic adenosine monophosphate and ATP in signal transduction in cardiac myocytes, potentiating an inward calcium current during the action potential. ${ }^{43}$ Preservation of ATP during hyperkalemic cardioplegic arrest by BDM may result in a potentiation of the $\beta$-adrenergic response due to this synergism between ATP and cyclic adenosine monophosphate.

The isolated myocyte model used in this study allows measurement of contractile function in a precisely controlled extracellular milieu so that direct influences of agents such as BDM can be determined. Examination of contractile properties of isolated myocytes has other distinct advantages, including the removal of both loading conditions and neurohormonal activity, which could potentially influence ventricular performance in vivo, and the ability to directly measure contractile function independent of the effects of alterations in coronary perfusion. Although the isolated myocyte model has the advantage of direct measurements of myocyte contractile function, it also has several limitations. The buffering influence of extracellular proteins, which may play an important role in vivo, is not present. Moreover, myocytes in the cardioplegic arrest groups were continuously exposed to an elevated potassium concentration without magnesium for the entire 2-hour incubation period, which differs from many intermittent multidose cardioplegia techniques used clinically. These limitations, however, also reveal the strength of the isolated myocyte model, because an examination can be made of the direct effects of cardioplegic arrest on the myocyte, the basic functional unit of the heart.

In summary, BDM prevented reductions in myocyte contractile function, $\beta$-adrenergic responsiveness, and cellular swelling after hypothermic, hyperkalemic cardioplegic arrest. This study provides for the first time direct evidence that the deleterious effects of cardioplegic arrest on myocyte contractile function, volume regulation, and active relaxation can be prevented with BDM supplementation of the cardioplegia solution. In light of the fact that BDM decreases intracellular calcium transients, these findings suggest that the mechanism of action of BDM supplementation during hyperkalemic cardioplegic arrest is improved calcium homeostasis. Although additional research is required, BDM supplementation holds clinical promise as an adjunct for myocardial protection with cardioplegic arrest.

\section{REFERENCES}

1. Gay WA. Potassium-induced cardioplegia: evolution and present status. Ann Thorac Surg 1989;48:441-3.

2. Gay WA, Ebert PA. Functional, metabolic and morphological effects of potassium-induced cardioplegia. Surgery 1973; 74:284-8.

3. Silverman NA, del Nido P, Krukenkamp I, Levitsky S. Biologic rationale for formulation of antegrade cardioplegic solutions. Cardiac Surg State Art Rev 1988;2(2):181-96.

4. Buckberg GD. Antegrade/retrograde blood cardioplegia to ensure cardioplegic distribution: operative techniques and objectives. J Cardiovasc Surg 1989;4:216-38.

5. Scheu SS, Blaustein MP. Sodium/calcium exchange and control of cell calcium and contractility in cardiac and vascular smooth muscles. In: Fozard HA, Haber E, Jennings RB, Katz AM, Morgan HE, eds. The heart and cardiovascular system. New York: Raven Press, 1992;903-44.

6. Cyran SE, Ditty SE, Baylen BG, Cheung J, LaNoue KF. Developmental differences in the response of cytosolic free calcium to potassium depolarization and cardioplegia in cardiac myocytes. J Mol Cell Cardiol 1992;24:1167-77.

7. Cyran SE, Phillips J, Ditty S, Baylen BG, Cheung J, LaNoue $K$. Developmental differences in cardiac myocyte calcium homeostasis after steady-state potassium depolarization: mechanisms and implications for cardioplegia. J Pediatr 1993;122:577-83.

8. Cavallo MJ, Dorman BH, Spinale FG, Roy RC. Myocyte contractile responsiveness following hypothermic hyperkale- 
mic cardioplegic arrest; disparity between exogenous calcium and $\beta$-adtenergic stimulation. Anesthesiology 1995;82:926-39.

9. Handy JR, Spinale FG, Mukherjee R, Crawford FA. Hypothermic potassium cardioplegia impairs myocyte recovery of contractility and inotropy. J THORAC CARDIOvaSC SuRG 1994;107:1050-8.

10. Roberts AJ, Spies SM, Sanders JH, et al. Serial assessment of left ventricular performance following coronary artery bypass grafting. J ThORAC CARDIOvasC SURG 1981;81:69-84.

11. Barry WH. Mechanical dysfunction of the heart during and after ischemia. Circ 1990;82:652-4.

12. Kihara Y, Grossman W, Morgan JP. Direct measurement of changes in intracellular calcium transients during hypoxia, ischemia and reperfusion of the intact mammalian heart. Circ Res 1989;65:1029-44.

13. Lee H, Mohabir R, Smith N, Franz M, Clusin W. Effect of ischemia on calcium-dependent fluorescence transients in rabbit hearts containing indo-1. Circulation 1988;78:1047-59.

14. Steenbergen C, Murphy E, London RE. Elevation in cytosolic free calcium concentration early in myocardial ischemia in perfused rat heart. Circ Res 1987;60:700-7.

15. Stringham JC, Paulsen KL, Southard JH, Fields BL, Belzer FO. Improved myocardial ischemic tolerance by contractile inhibition with 2,3 butanedione monoxime. Ann Thorac Surg 1992;54:852-60.

16. Stringham JC, Paulsen KL, Southard JH, Mentzer RM, Belzer FO. Prolonging myocardial preservation with a modified University of Wisconsin solution containing 2,3-butanedione monoxime and calcium. J THORAC CARDIOvasC SURG 1994;107:764-75.

17. Horiuti $\mathrm{K}$, Higguchi $\mathrm{H}$, Umazume $\mathrm{Y}$, Konishi M, Okazaki $\mathrm{O}$, Kurihara S. Mechanism of action of 2,3-butanedione 2-monoxime on contraction of frog skeletal muscle fibers. J Muscle Res Cell Motil 1988;9:156-64.

18. Gwathmey JK, Hajjar RJ, Solaro RJ. Contractile deactivation and uncoupling of crossbridges. Effects of 2,3-butanedione monoxime on mammalian myocardium. Circ Res 1991; 69:1280-92

19. Perreault CL, Mulieri LA, Alpert NR, Ransil BJ, Allen PD, Morgan JP. Cellular basis of negative inotropic effect of 2,3 Butanedione monoxime in human myocardium. Am J Physiol 1992;263:H503-10.

20. Steele DS, Smith GL. Effects of 2,3-butanedione monoxime on sarcoplasmic reticulum of saponin-treated rat cardiac muscle. Am J Physiol 1993;265:H1493-500.

21. Coulombe A, Lefevre IA, Deroubaix E, Thuringer D, Coraboeuf E. Effect of 2,3-butanedione 2 monoxime on slow inward and transient outward currents in rat ventricular myocytes. J Mol Cell Cardiol 1990;22:921-32.

22. Blanchard EM, Smith GL, Allen DL, Alpert NR. The effects of 2,3-butanedione monoxime on initial heat, tension and aequorin light output of ferret papillary muscles. Pfluegers Arch 1990;416:219-21.

23. Spinale FG, Mukherjee R, Fulbright BM, Hu J, Crawford FA, Zile MR. Contractile properties of isolated porcine ventricular myocytes. Cardiovasc Res 1993;27:304-11.

24. Ikenouchi $\mathrm{H}$, Zhao L, Barry WH. Effect of 2,3-butanedione monoxime on myocyte resting force during prolonged metabolic inhibition. Am J Physiol 1994;267:H419-30.

25. Spinale FG, Crawford FA, Hewett KW, Carabello BA. Ventricular failure and cellular remodeling with chronic supraventricular tachycardia. J THorac Cardiovasc Surg 1991:102:874-82

26. Quaife RA, Kohmoto D, Barry WH. Mechanisms of reoxygenation injury in cultured ventricular myocytes. Circulation 1991;83:566-77.

27. Fleckenstein A, Janke J, Doring HJ, Leader O. Myocardial fiber necrosis due to intracellular $\mathrm{Ca}^{2+}$ overload-a new principle in cardiac pathophysiology. In: Dhalla NS, ed. Recent advances in studies on cardiac structure and metabolism, vol 4. Baltimore: University Park, 1974:563-80.

28. Kimura M, Aviv A, Reeves JP. ${ }^{+}$-dependent $\mathrm{Na}^{+} / \mathrm{Ca}^{2+}$ exchange in human platelets. J Biol Chem 1993;268:6874-7.

29. Rayson BM. Calcium: a mediator of the cellular response to chronic $\mathrm{Na}^{+} / \mathrm{K}^{+}$-ATPase inhibition. J Biol Chem 1993;268: $8851-4$.

30. Tani M, Neely JR. Role of intracellular $\mathrm{Na}^{+}$in $\mathrm{Ca}^{2+}$ overload and depressed recovery of ventricular function of reperfused ischemic rat hearts. Circ Res 1989;65:1045-56.

31. Higuchi $\mathrm{H}$, Takemori $\mathrm{S}$. Butanedione monoxime suppresses contraction and ATPase activity of rabbit skeletal muscle. J Biochem 1989;105:638-43.

32. Weng ZC, Nicolosi AC, Detwiler PW, et al. Effects of crystalloid, blood and University of Wisconsin perfusates on weight, water content, and left ventricular compliance in an edema-prone isolated porcine heart model. J THORAC CARDIOVASC SURG 1992;103:504-13.

33. Bolli R. Mechanism of myocardial "stunning." Circulation 1990;82:723-38.

34. Lang F, Ritter M, Volk1 H, Haussinger D. The biological significance of cell volume. Rev Physiol Biochem 1993;16:4865.

35. Kato Y, Otani H, Tanaka K, Saito Y, Fukunaka M, Imamura H. Effect of cardioplegic preservation on intracellular calcium transients. Ann Thorac Surg 1991;52:979-86.

36. Lee SC, Dhalla NS. Subcellular calcium transport in failing hearts due to calcium deficiency and overload. Am J Physiol 1976;231:1159-65.

37. Dhalla NS, Das PK, Sharma GP. Subcellular basis of cardiac contractile failure. J Mol Cell Cardiol 1978;10:363-85.

38. Higuchi $\mathrm{H}$, Takemori $\mathrm{S}$. Butanedione monoxime suppresses contraction and ATPase activity of rabbit skeletal muscle. J Biochem 1989;105:638-43.

39. Schwinn DA, Leone BJ, Spahn DR, et al. Desensitization of myocardial $\beta$-adrenergic receptors during cardiopulmonary bypass: evidence of early uncoupling and late downregulation. Circulation 1991;84:2559-67.

40. Endoh M, Blinks JR. Actions of sympathomimetic amines on $\mathrm{Ca}^{2+}$ transients and contractions of rabbit myocardium: reciprocal changes in myofibrillar responsiveness to $\mathrm{Ca}^{2+}$ mediated through $\alpha$ and $\beta$-adrenoceptors. Circ Res 1988; 62:247-65.

41. Winegrad S. Regulation of cardiac contractile proteins: correlations between physiology and biochemistry. Circ Res 1984;55:565-74.

42. Jennings RB, Schaper J, Hill ML, Steenbergen C, Reimer KA. Effect of reperfusion late in the phase of reversible ischemic injury: changes in cell volume, electrolytes, metabolites, and ultrastructure. Circ Res 1985;56:262-78.

43. Zheng J, Christie A, DeYoung NB, Levy MN, Scarpa A. Synergism between cAMP and ATP in signal transduction in cardiac myocytes. Am J Physiol 1992;262:C128-35. 